\title{
Ideación Suicida en Adolescentes: Un Análisis Psicosocial
}

\section{Suicidal Ideation among Adolescents: A Psychosocial Analysis}

\author{
Juan Carlos Sánchez-Sosa \\ Universidad Autónoma de Nuevo León - México \\ Gonzalo Musitu \\ Universidad Pablo de Olavide - España
}

\author{
María Elena Villarreal-González \\ Universidad Autónoma de Nuevo León - México \\ Belen Martínez Ferrer \\ Universidad Pablo de Olavide - España
}

\begin{abstract}
Resumen. Se especificó y contrastó un modelo explicativo psicosocial de ideación suicida en adolescentes, mediante un estudio explicativo de tipo transversal. Se utilizó una muestra probabilística estratificada de 1285 estudiantes mexicanos de escolaridad media y media superior. Los instrumentos utilizados tenían índices adecuados de fiabilidad. Se contrastó el modelo con la técnica de ecuaciones estructurales utilizando el paquete estadístico EQS 6.1 que explicó el 33.10 \% de varianza explicada. Los resultados mostraron que el modelo obtuvo niveles adecuados de bondad de ajuste absoluto (RMSEA=.41; GFI=.953; AGFI=.939) y comparativo (CFI=.951; $\mathrm{IFI}=.958$; NFI=.925; NNFI=.943). Se concluyó que el Modelo Explicativo Psicosocial de Ideación Suicida (MEPIS) se ajustaba bien a los datos y era compatible con el modelo teórico propuesto, al integrar una estructura contextual que presentaba una relación indirecta y significativa del funcionamiento familiar y los problemas de integración escolar con la variable dependiente. Además, también se observó, una relación directa y significativa de la victimización escolar, sintomatología depresiva y conducta alimentaria de riesgo con la ideación suicida.
\end{abstract}

Palabras clave: adolescencia, escuela, factores psicosociales, familia, ideación suicida.

\begin{abstract}
This study tested a model of suicidal ideation in adolescents through a transverse type explanatory study. A stratified random sample of 1285 Mexican students from middle and high school was used. The questionnaires had an acceptable reliability index. The model is contrasted using the structural equations technique and the statistical package EQS 6.1, which explained the 33.10 of variance. The results showed satisfactory levels of absolute goodness of fit $(\mathrm{RMSEA}=.41$, GFI =. 953, AGFI =. 939) and comparative goodness of fit $(\mathrm{CFI}=.951$, IFI =. 958; NFI =. 925, NNFI =. 943). Finally, the Psychosocial Suicide Ideation Model (MEPIS, in Spanish) conforms well to the data and was compatible with the theoretical model proposed, in that it integrates a contextual structure showing a significant indirect relationship of family functioning and problems of school integration with the dependent variable. In addition, it was also observed that there was a direct and significant relation between school victimization, depressive symptoms, eating risk behavior and suicidal ideation.

Keywords: adolescence, family, psychosocial factors, school, suicidal ideation.
\end{abstract}

\section{Introducción}

El suicidio podría definirse como un proceso que se inicia con la ideación en sus diferentes expresiones (preocupación autodestructiva, planificación de un acto letal, deseo de muerte) discurre por el intento suicida y finaliza con el suicidio consumado. Por esta razón, se considera que la investigación en ideación suicida, así como la identificación de factores asociados a ésta, resulta de particular importancia en la prevención del suicidio (Paneth y Susser, 2002; Perez, 1999). En estudios recientes se subraya que el suicidio representa la tercera causa de muerte de adolescentes en el mundo (Suk, Van Mill, Vermeiren, Ruchkin, Schwab-Stone, Doreleijers y Deboutte 2009; World Health Organization, 2001). La Organización Mundial

La correspondencia sobre este artículo puede dirigirse a Juan Carlos Sánchez-Sosa. Facultad de Psicología. Universidad Autónoma de Nuevo León. Ave. Dr. Carlos Canseco \# 110 y Dr. Eduardo Aguirre Pequeño. Colonia Mitras Centro. C.P.64460, Monterrey Nuevo León. México.E-mail: juan.sanchezss@uanl.edu.mx de la Salud -OMS- (2006) informa que aproximadamente un millón de personas murieron por suicidio en el año 2000, y que las tasas de suicidio global han aumentado en un $60 \%$ en los últimos 45 años.

En el ámbito de la adolescencia, en la mayoría de los países de todo el mundo se está informando que las tasas de suicidio entre los adolescentes está aumentando de forma alaramante (OMS, 2006). En México, lugar donde se ha realizado este estudio, se ha constatado que entre 1990 y 2000 , la tasa de mortalidad por suicidios se duplicó en el grupo de 11 a 19 años con respecto al período anterior de 1980 a 1990, con un incremento todavía más marcado entre las mujeres (Puentes, López y Martínez, 2004). Además, el suicidio tiene un profundo impacto psicológico y social que afecta directamente a otras personas, fundamentalmen-

\footnotetext{
Agradecimientos: Esta investigación se ha elaborado en el marco del proyecto de investigación PSI 2008-01535/ PSIC "Violencia escolar: victimización y reputación social en la adolescencia" subvencionado por el Ministerio de Ciencia e Innovación de España.
} 
te las más próximas (Suk et al., 2009). La OMS en el 2000 , señala que un suicidio individual afecta profundamente al menos a otras seis personas y, en caso de ocurrir en una institución educativa o en el lugar de trabajo, el impacto es todavía mayor.

La ideación suicida es un fenómeno complejo en donde intervienen factores tanto personales, como familiares y escolares (Cheng, Tao, Riley, Kann, Ye, Tian, Tian, Hu y Che, 2009). En relación al contexto familiar, Lai y Shek (2009) en una investigación de 5557 estudiantes de secundaria de Hong Kong obtuvieron correlaciones significativas $(\mathrm{r}=-.460)$ entre funcionamiento familiar e ideación suicida. También, Van Renen y Wild (2008) constataron en una muestra de adolescentes que el grupo que mostraba mayor ideación suicida también tenía una menor comunicación y mayores conflictos con sus padres. En un estudio de prevalencia realizado en la Ciudad de México, Pérez, Rivera, Atienzo, de Castro, Leyva, y Chávez, (2010) concluyeron que los estudiantes que informaron que tenían un bajo apoyo familiar tenían un $69 \%$ más de probabilidad de presentar ideación suicida.

Además del contexto familiar, la escuela representa para el adolescente un contexto interactivo crucial en su desarrollo psicosocial (Estévez, Jiménez y Musitu, 2007; Musitu, Jiménez y Estévez, 2009). En un trabajo reciente, Sánchez-Sosa (2009) observó una relación negativa y significativa entre el ajuste escolar y la ideación suicida. También, Pérez et al. (2010) constataron que los adolescentes con poco reconocimiento escolar son más proclives a manifestar ideación suicida, y, en la misma línea conceptual, Bonanno y Hymel (2010) observaron mediante un análisis de regresión que la victimización escolar es un factor predictivo de la ideación suicida.

Junto con los contextos familiar y escolar el factor personal o psicológico representa el otro escenario en donde las variables que lo representan tienen una mayor relación con la ideación suicida que las variables que representan los escenarios familiar y escolar (Sánchez-Sosa, 2009; Sun, Hui y Watkins 2006). Por ejemplo, se ha observado en numerosas investigaciones que la depresión es la variable más relacionada con la ideación suicida (Au, Lau y Lee, 2009; Garlow, Rosenberg, Moore, Haas, Koestner, Hendin, y Nemeroff, 2008; McLaren y Challis, 2009). En este sentido, Hintikka, Koivumaa, Lehto, Tolmunen, Honkalampi, Haatainen, y Viinamaki, (2009) concluyeron en un interesante trabajo que un estado de ánimo depresivo se debe considerar como una condición previa necesaria para que surja la ideación suicida.

En relación con las conductas alimentarias de riesgo, en estudios recientes se han obtenido relaciones significativas entre variables asociadas a problemas alimenticios y la ideación suicida (Sánchez-Sosa, Villarreal, Musitu, 2010). Recientemente, Goldney, Dunn, Air, Dal Grande y Taylor (2009) realizaron un estudio para determinar la relación entre índice de masa corporal, salud mental e ideación suicida y concluyeron que no existe relación entre valores altos de índice de masa corporal e ideación suicida. En una investigación con adolescentes coreanos, Don-Sik, Youngtae, Sung-Il y In-Sook (2009) observaron una relación significativa entre valores bajos de índice de masa corporal, conductas alimentarias de riesgo y la ideación suicida. Estos hallazgos sugieren que más que una relación con índices antropométricos, la ideación suicida está asociada a trastornos de conducta alimentaria.

Entre las variables psicológicas, se ha demostrado que la autoestima es una variable particularmente importante para explicar la ideación suicida. En este sentido, Miranda, Cubillas, Román y Valdez (2009) encontraron que el grupo con ideación suicida presentaba una autoestima significativamente más baja que el grupo sin ideación. Por otra parte, Au, Lau y Lee (2009) observaron correlaciones significativas con medidas de autoconcepto social.

Es en esta multiplicidad y complejidad de factores y contextos relacionados con la ideación suicida donde se enmarca el presente trabajo. El objetivo es contrastar un modelo explicativo hipotético en adolescentes escolarizados en el que se analizan los efectos directos e indirectos en la ideación suicida a partir de variables personales (autoestima social, sintomatología depresiva, conducta alimentaria de riesgo) y variables contextuales familiares (funcionamiento familiar) y escolares (ajuste y victimización escolar). El interés de este objetivo radica en el hecho de que en la literatura sobre el tema existen muy pocas investigaciones en las que se hayan analizado la influencia conjunta de diversos contextos y variables personales (Sun, Hui y Watkins 2006; Yoder y Hoyt, 2005). Se considera que con este análisis se coadyuvará en la explicación de este problema y servirá de base para el diseño de programas de prevención de la ideación suicida.

La representación grafica del modelo teórico denominado Modelo Explicativo Psicosocial de Ideación Suicida (MEPIS), se presenta en la Figura 1.

\section{Método}

\section{Participantes}

La muestra estuvo conformada por 1285 estudiantes mexicanos de escolaridad media y media superior pertenecientes a cuatro escuelas públicas: dos de educación secundaria $(n=634)$ y dos preuniversitaria $(n=$ 651). Los adolescentes de la muestra tenían edades comprendidas entre los 12 y los 18 años, con una media de edad de 15.07 y una desviación estándar de 1.5. El $36.1 \%$ de los participantes pertenecían a la adolescencia temprana (12-14); el 60.7\% a la adolescencia media (15-17) y, finalmente, el $3.2 \%$ a la adolescencia tardía (18-21). La muestra presenta porcentajes equivalentes en género con 645 mujeres y 640 hombres. 
Figura 1. Modelo hipotético de ideación suicida

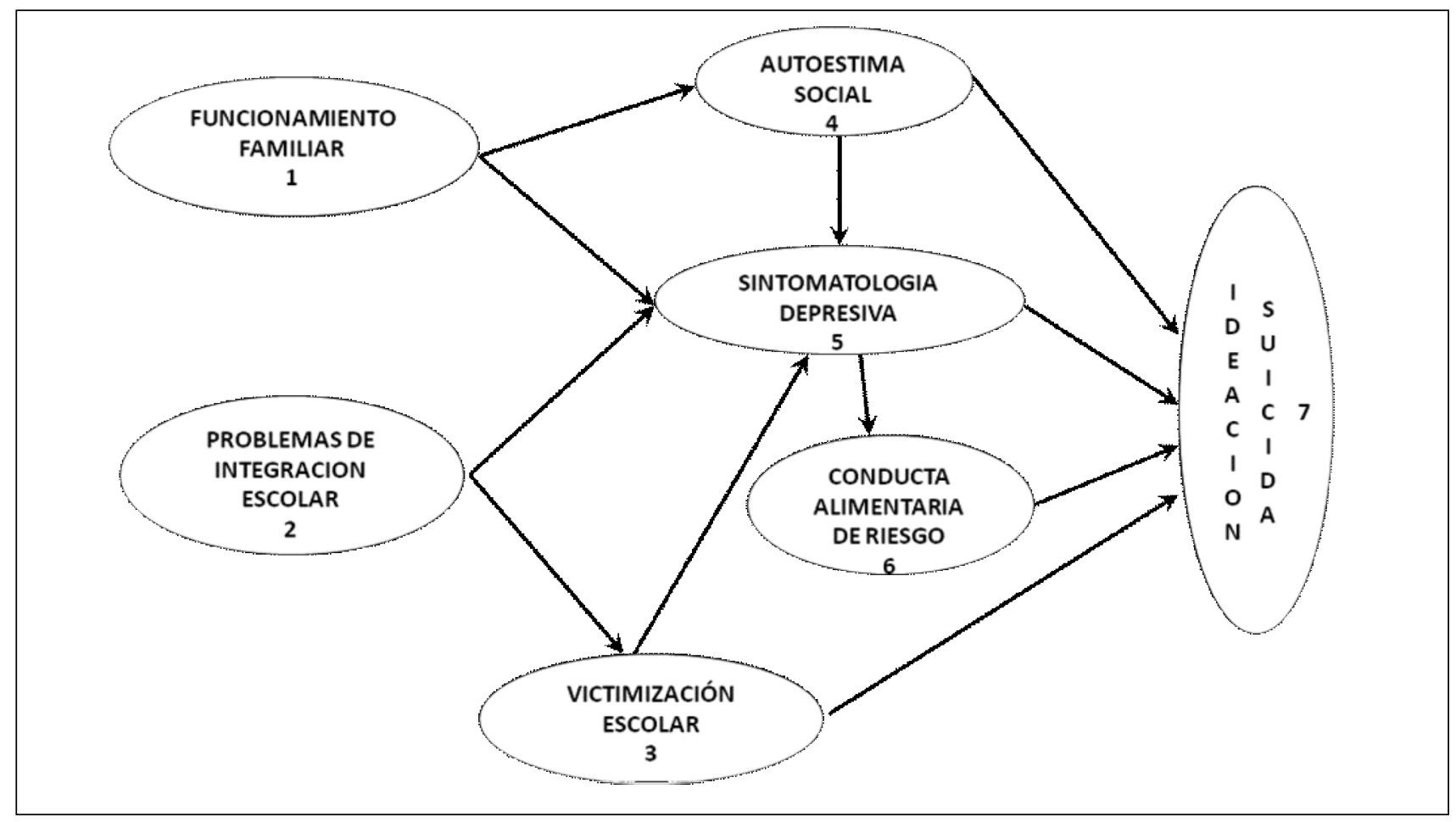

\section{Instrumentos}

Las variables relacionadas con la ideación suicida se aglutinaron en dos grupos: variables contextuales y variables personales. Las variables contextuales fueron: funcionamiento familiar, ajuste escolar y victimización escolar. Las escalas fueron las siguientes:

Cuestionario de Funcionamiento Familiar, APGAR Familiar, de Smilkstein, Ashworth y Montano (1982). Esta escala fue adaptada al castellano por Bellon, Luna y Lardelli (1996). La escala original mostró una consistencia interna de $\alpha=.84$. Se obtuvo el mismo - en una muestra chilena (Caqueo y Lemos, 2008). Es una escala unifactorial tipo likert de tres opciones de respuesta (casi nunca, a veces, casi siempre) que consta de 5 reactivos y proporciona un nivel general de funcionamiento familiar (por ejemplo, «¿Estás satisfecho con la ayuda que recibes de tu familia cuando tienes algún problema?», «¿Sientes que tu familia te quiere?»). En el presente estudio la escala obtuvo una consistencia interna de $\alpha=.79$. Este cuestionario se ha utilizado en diversos estudios, para la valoración familiar en casos de alcoholismo, sida, depresión y embarazos en adolescentes (Rangel, Valerio, Patiño y García, 2004). Para su codificación se estiman valores de $>6$ como funcional $\mathrm{y}<6$ como disfuncional. En algunos casos, se valora de 0 a 3 como disfunción grave y de 3 a 6 como leve y la funcionalidad familiar se contemplan las puntuaciones de 7 a 10. En la validación española de Bellon, Delgado, Luna y Lardelli (1996) la fiabilidad test-retest fue de .75 . En cuanto a la validez, en el trabajo original se obtuvo un alto grado de correlación (.80) con el Pless-Satterwhite Family Function Índex. El análisis factorial de la validación española reveló la existencia de un único factor en la escala integrado por los 5 ítems, que explicó el $61.9 \%$ de la varianza.

Escala breve de ajuste escolar de Moral, SánchezSosa y Villarreal-González (2010). Esta escala tiene un Alpha de Cronbach de .79. Consta de 10 ítems con un formato tipo likert con un rango de 6 puntos. Cinco ítems están redactados en sentido inverso $(6,7,8,9$ y 10). El rango de la escala va de 10 a 60 . En un estudio reciente, Moral, Sanchez-Sosa y Villarreal-González (2010) al factorizar por Componentes Principales, con base en el criterio Kaiser, definieron tres factores que explican el 59.597\% de la varianza total: 1. Problemas de integración escolar: Está constituida por cinco reactivos que reflejan problemas de adaptación al medio escolar («creo que la escuela es aburrida»); 2. Rendimiento escolar: Está constituida por tres reactivos («disfruto realizando mis tareas escolares») y, 3 . Expectativa académica: Está constituida por dos reactivos («Estoy interesado/a en asistir a la Universidad $»$ ). Tiene un rango de respuesta de 1 a 6 (que van de completamente en desacuerdo a completamente de acuerdo). En cuanto a validez concurrente, la escala presenta una correlación directa con comunicación familiar positiva y, a su vez, inversa, con comunicación familiar negativa e ideación suicida. Para esta investigación se utilizo la subescala de problemas de integración escolar con una consistencia interna en este estudio de $\alpha=.84$. 
Escala de conductas predelictivas de Rubini y Pombeni (1992). Consta de 23 reactivos dicotómicos con una consistencia interna de $\alpha=.87$. Para el presente estudio se utilizó la adaptación al castellano (Cava, 2006) que consta de 19 reactivos tipo likert de cinco opciones de respuesta (nunca, casi nunca, algunas veces, bastantes veces y muchas veces). La escala presenta dos factores: el primero de ellos mide conductas agresivas o predelictivas («he pintado o dañado las paredes de la escuela», «he insultado a compañeros/as de clase») y, el segundo, victimización («Algún compañero me insultó o me pegó», «Se burlaron de mí en clase o me hicieron daño»). La escala global de la adaptación española reporta una fiabilidad $\alpha=.92$ que es similar a la obtenida en el presente estudio $\alpha=.93$ ). Para la presente investigación se utilizó la subescala de victimización que consta de seis reactivos y un coeficiente Alpha de Cronbach de .84. La dimensión victimización muestra correlaciones positivas con la presencia de sintomatología depresiva y de estrés percibido. La dimensión conducta violeta correlaciona negativamente con la actitud positiva hacia la autoridad, la autoestima familiar y el apoyo familiar.

Para medir las variables personales de sintomatología depresiva, autoestima social, y conducta alimentaria de riesgo se utilizaron las siguientes escalas.

Cuestionario de evaluación de la sintomatología depresiva de Radloff (1977). La versión original muestra una consistencia interna de $\alpha=.85$ con una muestra aleatoria, y $\alpha=.90$ con una muestra clínica. La fiabilidad de la escala global de la adaptación Española es de $\alpha=.83$. La escala original está integrada por 20 reactivos, con un rango de 0 a 3 y un recorrido de 0 a 60. Los reactivos de la escala fueron seleccionados por Radloff de otras escalas de depresión. De los 20 reactivos, 16 están redactados de forma directa $(1,2,3,5$, $6,7,9,10,11,13,14,15,17,18,19,20)$ y 4 en forma inversa $(4,8,12,16)$. En el instrumento adaptado, la escala tipo likert se amplía de 4 a 5 posibilidades de respuesta (nunca, pocas veces, algunas veces, muchas veces, siempre). Por lo que a diferencia de la escala original el recorrido de la adaptación va de 20 a 100 . Este instrumento, evalúa la sintomatología asociada normalmente con la depresión, pero no evalúa la depresión en sí misma por ejemplo, (« Creí que mi vida había sido un fracaso», «Me sentí solo/a», «Tenía ganas de 1lorar»). Tanto la adaptación al castellano como la escala original reportan una estructura mono factorial. La estimación de la escala se obtiene mediante la suma de todos los reactivos por que se tienen que invertir los valores de los reactivos redactados en sentido inverso $(4,8,12,16)$. A mayor puntaje mayor ánimo depresivo. Para el presente estudio la escala mostró una coeficiente Alpha de Cronbach de .82 .

Cuestionario de evaluación de autoestima en adolescentes AF5 de García y Musitu (1999). Es un instrumento de 30 reactivos formulados en términos positivos y negativos que miden el autoconcepto de los suje- tos en cinco dimensiones: académica («mis profesores me estiman»), familiar («me siento querido por mis padres»), física («Soy una persona atractiva»), social («soy una persona amigable») y emocional («me asusto con facilidad»). La estructura pentadimensional se fundamenta en el modelo teórico de Shavelson, Hubner y Stanton (1976), quienes, entre otras características como la organización jerárquica a partir de una dimensión general, consideran que el autoconcepto presenta diversos aspectos relacionados (no ortogonales) pero distinguibles, que pueden encontrarse diferencialmente relacionados con diversas áreas del comportamiento humano (Musitu, García y Gutiérrez, 1994). Cuenta con un rango de respuestas que oscila entre 1 (nunca) a 5 (siempre). A mayor puntuación en cada uno de los factores mencionados, corresponde mayor autoconcepto en dicha dimensión. La versión original reporta una consistencia interna de $\alpha=.81$ para la escala general. En cuanto a su validez, la escala discrimina entre hombres y mujeres; los hombres muestran mayor nivel de autoestima emocional y física que las mujeres, mientras que éstas muestran mayor nivel de autoestima académica. En relación con la autoestima académica y física los adolescentes de 1214 años expresan, mayores niveles que los adolescentes de 15-17 y 18-20. Todas las dimensiones de la autoestima correlacionan positivamente con la dimensión de socialización de apoyo, y negativamente con las de coerción, sobreprotección y reprobación (Musitu, García y Gutiérrez, 1994). En este estudio se utilizo la subescala de autoestima social que consta de cinco reactivos. La consistencia interna obtenida para esta subescala es de $=.78$ y para la escala global $\alpha=.85$.

Cuestionario breve de conducta alimentaria de riesgo de Unikel, Bojorquez y Carreño (2004). El cuestionario fue elaborado a partir de los criterios diagnósticos del DSM-IV. Consta de 10 preguntas sobre preocupación por engordar, práctica de atracones, sensación de falta de control al comer y conductas alimentarias de tipo restrictivo (dietas, ayunos, ejercicio y uso de pastillas para bajar de peso) y purgativo (vómito autoinducido, uso de laxantes y de diuréticos). La escala consta de 4 opciones de respuesta: nunca o casi nunca, algunas veces, frecuentemente (dos veces en una semana) o muy frecuentemente (más de dos veces en una semana). La mayor puntuación en el cuestionario corresponde a mayor cantidad de anomalías en la conducta alimentaria. Presenta una estructura factorial de tres dimensiones: Conducta Purgativa («he vomitado después de comer para tratar de bajar de peso»), Conductas Compensatorias («he hecho dietas para tratar de bajar de peso») y Atracones («en ocasiones he comido demasiado, me he atascado de comida»). La consistencia interna de la escala original es de $\alpha=.83$. En el presente estudio se obtuvo una consistencia interna de $\alpha=.82$.

Escala de Ideación Suicida de Roberts (1980). Se utilizo la adaptación para población mexicana de Mariño, Medina, Chaparro y González (1993). La 
Escala consta de cuatro reactivos: «no podía seguir adelante», «tenía pensamientos sobre la muerte», «sentía que mi familia estaría mejor si yo estuviera muerto», $\mathrm{y}$ «pensé en matarme». Las opciones de respuesta permiten conocer la ocurrencia de los síntomas en la última semana: $1=$ "0 días"; $2=$ " $1-2$ días"; $3=$ "3-4 días", y 4= "5-7 días". El rango de la escala varía de 4 a 16. Todos los reactivos están redactados en sentido directo y la puntuación en la escala se obtiene por la suma simple de reactivos. A mayor puntaje mayor ideación suicida. La fiabilidad de la escala en adolescentes mexicanos, varía en diversos estudios de $\alpha=.78$; $\alpha \cdot=.81 ; \alpha=.83 ; \alpha=.88$. En esta investigación el coeficiente Alpha de Cronbach fue de $\alpha=.84$.

\section{Procedimiento}

Se aplicaron los cuestionarios, todos de autoinforme, a cuatro centros educativos: dos escuelas preuniversitarias y dos escuelas secundarias de dos municipios conurbados (varios núcleos urbanos inicialmente independientes y contiguos, que al crecer acaban formando una unidad funcional) del estado de Nuevo León, México. Se administraron 1285 cuestionarios (651 en nivel de preuniversitario y 634 en secundaria). En cada aula, se entregaron a cada alumno los cuestionarios correspondientes y se asignaron dos encuestadores para cada grupo. Se solicitó la colaboración voluntaria y se les garantizó la confidencialidad y el anonimato de las respuestas. Para evitar el efecto de fatiga y la posibilidad de obtener respuestas falseadas debido a la fatiga se administró la batería de instrumentos en dos momentos temporales con un intervalo de 2 días.

\section{Análisis de Datos}

Para el análisis estadístico de los datos se utilizaron los paquetes estadísticos SPSS15.0 y EQS 6.0 (Bentler, 1989). En el análisis de las variables, el nivel de significación estadística se fijó en .05. Se utilizó el Modelo de Ecuaciones Estructurales (SEM) con la finalidad de probar el modelo teórico de ideación suicida. Debido a la desviación de la multinormalidad de los datos se utilizaron estimadores robustos para determinar la bondad de ajuste del modelo y la significación estadística de los coeficientes. Para el contraste del modelo estructural se contemplaron tres índices de bondad de ajuste absoluto: 1) el error medio cuadrático de aproximación a valores de la población (RMSEA) cuyo valor ideal es $\leq .5$; 2) el índice de bondad de ajuste de Joreskog (GFI) el cual se interpreta como una proporción de varianza explicada análoga a $\mathrm{R} 2$ en regresión múltiple, por lo que un GFI de 1.0 indicaría ajuste perfecto lo cual significa que el modelo explica el $100 \%$ de la varianza en los datos observados. Cuanto más próximo a uno sea el valor, mejor bondad de ajuste en este indicador. El criterio más comúnmente aceptado es de $\geq .90 ; 3)$ el índice de bondad ajustado de Joreskog (AGFI) que ajusta el GFI tomando en cuenta los grados de libertad en el modelo a prueba. El criterio que se asigna regularmente a este indicador es $\geq .90$ (Tabla 1).

Asimismo se tomaron en cuenta cuatro índices de ajuste comparativo los cuales contrastan un modelo hipotetizado con el modelo de independencia que por definición tiene el peor ajuste. El modelo de independencia o modelo nulo es aquel en donde todas las correlaciones son próximas a cero, siendo el modelo saturado el otro lado del continuo (correlaciones perfectas). Los índices de ajuste comparativo tomados en cuenta son: 1) el índice de ajuste normado de BentlerBonett (NFI) se interpreta como un porcentaje de incremento en la bondad de ajuste sobre el modelo nulo. Por lo que un valor de 90 implica que el modelo hipotetizado ajusta $90 \%$ mejor que el nulo; 2) el índice no normado de Bentler-Bonett (NNFI) toma valores inferiores menores a cero y se considera que con puntuaciones superiores a .90 se asume un adecuado ajuste; 3) el índice comparativo de ajuste de Bentler (CFI) asume valores entre 0 y 1 y la regla práctica para el CFI es que valores de .90 o mayores son indicativos de ajuste razonable; 4) índice de ajuste de incremento de Bollen (IFI) que reintroduce un factor de escala para que los valores se mantengan en el rango de 0 a 1 . Los valores comparativamente más altos que otros indican mejor ajuste.

Tabla 1. Bondad de ajuste Modelo Explicativo de Ideación Suicida

\begin{tabular}{|c|c|c|c|}
\hline \multirow[b]{2}{*}{ Índices de ajuste } & \multicolumn{2}{|c|}{ Valores } & \multirow{2}{*}{$\begin{array}{c}\text { Modelo } \\
\text { Explicativo }\end{array}$} \\
\hline & $\begin{array}{c}\text { No } \\
\text { Adecuado }\end{array}$ & Adecuado & \\
\hline Error cuadrático medio de aproximación (RMSEA) & $>.099$ & $\leq .05$ & .041 \\
\hline Índice de bondad de ajuste de Joreskog (GFI) & $<.85$ & $\geq .95$ & .953 \\
\hline Índice de bondad ajustado de Joreskog (AGFI) & $<.80$ & $\geq .90$ & .939 \\
\hline Índice de ajuste normado de Bentler-Bonett (NFI) & $<.80$ & $\geq .90$ & .925 \\
\hline Índice de ajuste no normado de Bentler-Bonett (NNFI) & $<.85$ & $\geq .95$ & .943 \\
\hline Índice comparativo de ajuste de Bentler (CFI) & $<.85$ & $\geq .95$ & .951 \\
\hline Índice de Ajuste de Incremento o $\varnothing$ de Bollen (IFI) & $<.80$ & $\geq .90$ & .952 \\
\hline
\end{tabular}




\section{Resultados}

En la Tabla 2 se presenta la matriz de correlaciones de las variables. El análisis de correlación confirma que existen correlaciones estadísticamente significativas entre la mayoría de las variables del modelo. Asimismo, se observan correlaciones significativas y positivas de la variable dependiente con las siguientes variables: Sintomatología depresiva $(r=.511 ; p=$ $.001)$, Conducta alimentaria de riesgo $(r=.271 ; p=$ $.001)$, Victimización $(r=.262 ; p=.001)$ y Problemas de ajuste escolar $(r=.218 ; p=.001)$; y correlaciones significativas y negativas con el funcionamiento familiar $(r=-.314 ; p=.001)$ y con la autoestima social $(r$ $=-.188 ; p=.001)$.

Mediante la utilización de la técnica de ecuaciones estructurales se contrastó el modelo explicativo previamente especificado para analizar la influencia directa e indirecta de factores contextuales y personales en la ideación suicida El modelo propuesto presenta un buen ajuste a los datos tanto en los índices de ajuste comparativo $(\mathrm{CFI}=.951 ; \mathrm{IFI}=.958 ; \mathrm{NFI}=.925 ; \mathrm{NNFI}=$ .943), como en los índices de ajuste absoluto (GFI = .953; AGFI = .939; RMSEA $=.041$ ).

En la figura 2 se muestra el modelo estructural contrastado el cual explica un $33.10 \%$ de la varianza. Los resultados confirman una relación indirecta de las variables familiares y escolares con la ideación suicida. Asimismo, las variables personales de sintomatología depresiva, conducta alimentaria de riesgo y victimización escolar muestran efectos directos con la variable dependiente, la ideación suicida. Sin embargo,

Tabla 2. Matriz de correlación entre las variables del Modelo Explicativo Psicosocial de Ideación Suicida

\begin{tabular}{|c|c|c|c|c|c|c|c|}
\hline & $\begin{array}{l}\text { Ideación } \\
\text { suicida }\end{array}$ & $\begin{array}{c}\text { Sint. } \\
\text { depresiva }\end{array}$ & Victimización & Car. & $\begin{array}{l}\text { A.E. } \\
\text { social }\end{array}$ & $\begin{array}{l}\text { Prob. Aj. } \\
\text { escolar }\end{array}$ & $\begin{array}{l}\text { Func. } \\
\text { familiar }\end{array}$ \\
\hline Ideación suicida & 1 & & & & & & \\
\hline Sint. depresiva & $.511(* *)$ & 1 & & & & & \\
\hline Victimización & $.262(* *)$ & $.281(* *)$ & 1 & & & & \\
\hline Cond. alimentaria riesgo & $.271(* *)$ & $.373(* *)$ & $.240(* *)$ & 1 & & & \\
\hline Autoestima social & $-.188(* *)$ & $-.234(* *)$ & $-.161(* *)$ & -.038 & 1 & & \\
\hline Prob. ajuste escolar & $.218(* *)$ & $.252(* *)$ & $.382(* *)$ & $.196(* *)$ & $-.266(* *)$ & 1 & \\
\hline Func. familiar & $-.314(* *)$ & $-.382(* *)$ & $-.192(* *)$ & $-.168(* *)$ & $.256(* *)$ & $-.161(* *)$ & 1 \\
\hline
\end{tabular}

Figura 2. Modelo Explicativo Psicosocial de Ideación Suicida

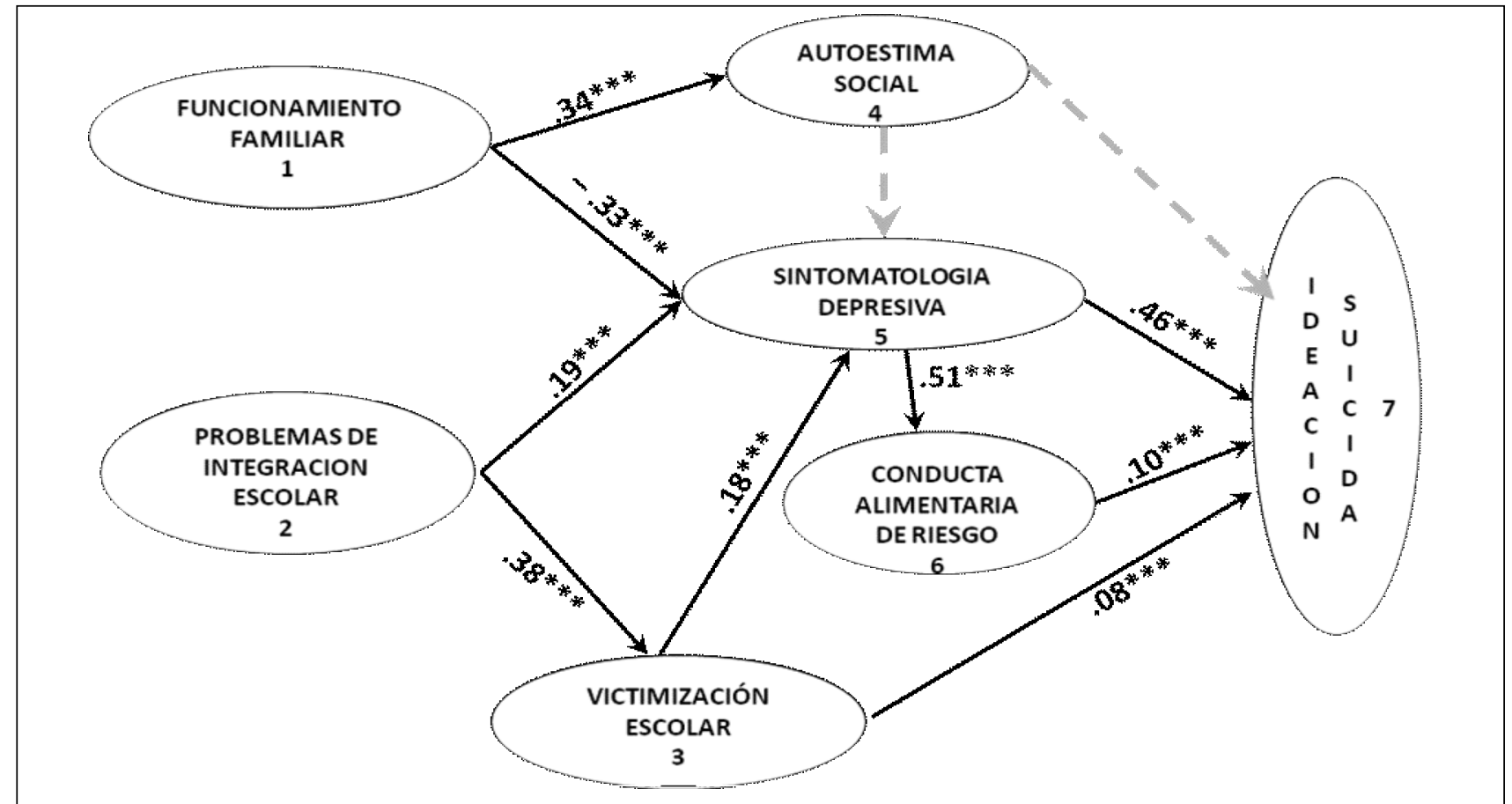

Nota. Las líneas continuas representan relaciones significativas entre las variables. La significación de las relaciones se ha determinado a partir del error estándar. ${ }^{* * *} p<0.001$. Las líneas discontinuas representan relaciones no significativas. 
la relación tanto directa como indirecta de la autoestima social con la ideación suicida no resultó significativa.

Se puede observar el efecto indirecto de las variables contextuales -funcionamiento familiar y problemas de integración escolar- con la variable dependiente, en donde el funcionamiento el familiar y los problemas de integración escolar tienen una relación directa con la sintomatología depresiva $(\beta=-.33 ; \beta=.19)$. A su vez, la sintomatología depresiva es la variable que mejor explica la ideación suicida al presentar una relación directa y positiva $(\beta=.46)$ y una relación indirecta a través del efecto directo con la conducta alimentaria de riesgo $(\beta=.51)$. Esta conducta, muestra también una asociación directa y positiva con la variable dependiente, la ideación suicida $(\beta=.10)$. El contexto escolar presenta una segunda trayectoria de relaciones indirectas y directas al observarse una relación directa y positiva de los problemas de integración escolar y la victimización. Esta variable, a su vez, presenta una doble relación con la ideación suicida: una relación indirecta a través de la sintomatología depresiva $(\beta=.18)$ y una relación directa y positiva $(\beta=.08)$.

\section{Discusión}

La ideación suicida se considera como un trastorno, como el inicio o primera etapa de un continuo que lleva al individuo a consumar el suicidio. La literatura disponible sobre el tema, al destacar su carácter multifactorial, pone de manifiesto la necesidad de plantear modelos explicativos que coadyuven a la prevención de este problema, que deberá de ser considerado no como causa o síntoma sino más bien como corolario de una serie de factores de riesgo que potencian el desarrollo de conductas desadaptativas en los adolescentes y que a su vez propician la ideación suicida.

En el presente trabajo, se han estudiado las relaciones entre variables contextuales y personales con la ideación suicida. Los resultados obtenidos al contrastar empíricamente el Modelo Explicativo Psicosocial de Ideación Suicida (MEPIS), presentan una trayectoria de relaciones directas e indirectas con la ideación suicida que implica variables contextuales y personales. Este modelo nos muestra que el buen funcionamiento familiar y una adecuada integración escolar pueden considerarse como factores protectores indirectos de la ideación suicida. El funcionamiento familiar se relaciona positivamente con la autoestima social y, negativamente, con la sintomatología depresiva y los problemas de integración escolar se relacionan positivamente con la sintomatología depresiva y con la victimización escolar, dimensiones éstas que tienen una relación directa con la ideación suicida. Se podría decir que un buen ajuste en los adolescentes reduciría el riesgo de ser victimizados, de padecer sintomatologías depresivas y llevar a cabo conductas alimentarias de riesgo y de tener ideaciones suicidas. Estos resultados son coherentes con los obtenidos por Villalobos (2009) en el sentido de que existen relaciones entre diferentes variables de riesgo y de protección con las conductas suicidas en general, y la ideación suicida en particular.

La trayectoria especificada y confirmada empíricamente en este estudio que señala a la sintomatología depresiva como la variable que mejor predice la ideación suicida concuerda con los datos obtenidos por diversos investigadores (Konick y Gutierrez, 2005; Park, Ryu, Han, Kwon, Kim, Kang, Yoon, Cheon y Shin, 2010) Otro aspecto en el que se concuerda con el trabajo de estos investigadores es en lo referente a la varianza explicada, ya que mientras las investigaciones citadas informan de una varianza explicada de $39 \%$ el modelo que en este trabajo hemos contrastado explica el $33.10 \%$.

La relación directa estimada entre la autoestima social y la ideación suicida que en el contraste empírico no fue significativa, concuerda con los hallazgos obtenidos por Jiménez, Mondragón y González (2007) quienes constataron que la autoestima no se relaciona significativamente con la ideación suicida. Sin embargo, la evidencia empírica con respecto a la relación entre autoestima e ideación suicida es controvertida ya que en un estudio de Yoder y Hoyt (2005) se detectó una relación directa y significativa entre estas variables. Asimismo, Wilburn y Smith (2005) proponían que una baja autoestima predispone al adolescente a la depresión y por ende a las ideas suicidas. En relación a lo señalado por estos autores, la relación estimada en el MEPIS respecto a la autoestima social y la sintomatología depresiva tampoco fue significativa. Como una alternativa que ayude a dilucidar la controversia entre autoestima e ideación suicida, proponemos que futuras investigaciones contemplen dicha relación considerando otras dimensiones de la autoestima como la emocional, física, familiar y educativa con la finalidad de proporcionar mayor especificidad y claridad en el análisis de estas variables.

El Modelo Explicativo Psicosocial de Ideación Suicida propuesto en esta investigación en el que se integran factores contextuales y personales se encuentra en la misma línea que en el modelo estructural propuesto por Sun, Hui y Watkins (2006), quienes observaron una relación directa de variables contextuales con la autoestima la cual, a su vez, tenía una relación directa con la depresión que finalmente predecía a la ideación suicida.

La diversidad de variables que se relacionan directamente con la ideación suicida (sintomatología depresiva, victimización escolar y conducta alimentaria de riesgo) es probablemente el hallazgo a destacar de la presente investigación, en la medida en que a diferencia de otros modelos explicativos en los que se informa de sólo relaciones directas y significativas de la depresión (Sun, Hui y Watkins 2006) o de otras variables relacionadas con el factor emocional, como la des- 
esperanza (Rud, 1990; Konick y Gutiérrez, 2005; Villalobos, 2009), el modelo que proponemos muestra la idea en gran parte defendida por la comunidad internacional de que la ideación suicida es un fenómeno multifactorial y que es en esta dirección por donde hay que caminar en los futuros trabajos científicos (Cheng, et al. 2009).

Con esta investigación podríamos afirmar que el MEPIS se ajusta bien a los datos y es compatible con el modelo teórico propuesto, al integrar una estructura contextual que presenta una relación indirecta y significativa del funcionamiento familiar y los problemas de integración escolar con la variable dependiente. Asimismo, muestra una relación directa y significativa de la victimización escolar, sintomatología depresiva y conducta alimentaria de riesgo con la ideación suicida.

Un resultado que nos parece de interés resaltar es la relación directa y significativa de la victimización escolar y la conducta alimentaria de riesgo con la ideación suicida porque, consideramos, que enriquece muy substantivamente lo hasta ahora planteado por la Comunidad Científica Internacional, en el sentido de que trascendemos las variables de tipo emocional para adentrarnos en otros escenarios en los que participa activamente el adolescente, como la familia (funcionamiento y comunicación), escolares (ajuste y clima), además del trastorno alimentario y la sintomatología depresiva, ésta última más comúnmente utilizada para la explicación de la ideación suicida.

Finalmente, y debido al carácter trasversal y correlacional del estudio, cabe aclarar que las relaciones expresadas no son de tipo causal (Paneth y Susser, 2002), por lo que los resultados obtenidos en este trabajo deben interpretarse con cautela. Pese a estas limitaciones, creemos que los hallazgos aquí expuestos pueden orientar futuras investigaciones que además de replicar las relaciones aquí analizadas, integren variables con la finalidad de construir modelos con mayor poder heurístico que permita el diseño de programas de prevención e intervención en ideación suicida.

\section{Referencias}

Au, A., Lau, S. y Lee, M. (2009). Suicide ideation and depression: the moderation effects of family cohesion and social self-concept. Adolescence, 44, 851868.

Bentler, P. (1989). EQS. Structural Equations Program Manual. Los Angeles: BMPD Statistical Software Inc.

Bronfenbrenner, U. (2002). La Ecología del Desarrollo Humano. Barcelona: Paidos.

Caqueo, A. y Lemos, S. (2008). Calidad de vida y funcionamiento familiar de pacientes con esquizofrenia en una comunidad latinoamericana. Psicothema, 20 , 577-582.
Cava, M., Musitu, G. y Murgui, S. (2006). Familia y violencia escolar: el rol mediador de la autoestima y la actitud hacia la autoridad institucional. Psicothema, 18, 367-373.

Cheng, Y., Tao, M., Riley, L., Kann, L., Ye, L., Tian, X., Tian, B., Hu, J. y Che, D. (2009). Protective factors relating to decreased risks of adolescent suicidal behavior. Child: care, health and development, 35, 313-322. doi:10.1111/j.1365-2214.2009.009 55.X

Dies, L., Azevedo, R., Jansen, K., Peretti, R., Lessa, B. y Tavares, R. (2010). Suicidal ideation in adolescents aged 11 to 15 years: prevalence and associated factors. Revista Brasileira de Psiquiatria, 32, 37-41.

Don-Sik, K., Youngtae, C., Sung-Il, C. y In-Sook, L. (2009). Body Weight Perception, Unhealthy Weight Control Behaviors, and Suicidal Ideation Among Korean Adolescents. Journal of School Health, 79, 585-592.

Estévez, E., Jiménez, T. y Musitu, G. (2007). Relaciones entre padres e hijos adolescentes. Valencia: Nau Llibres.

Fouce, J.G. y Fossoul, M.M. (2007). Prevenir en otra onda. Intervención Psicosocial, 16, 229-259.

García, F. y Musitu, G. (1999). Autoconcepto Forma 5. Madrid: TEA.

Garlow, S., Rosenberg, J., Moore, J., Haas, A., Koestner, B., Hendin, H. y Nemeroff, C. (2008). Depression, desperation, and suicidal ideation in college students: results from the American foundation for suicide prevention college screening project at emory University. Depression And Anxiety 25, 482-488. DOI 10.1002/da.20321.

Goldney, R., Dunn, K., Air, T., Dal Grande, E. y Taylor, A. (2009). Relationships between body mass index, mental health, and suicidal ideation: population perspective using two methods. Australian and New Zealand Journal of Psychiatry, 43, 652-658.

Hintikka, J., Koivumaa-Honkanen, H., Lehto, S., Tolmunen, T., Honkalampi, K., Haatainen, K. y Viinamaki, H. (2009). Are factors associated with suicidal ideation true risk factors? A 3-year prospective follow-up study in a general population. Sociological Psychiatric Epidemiology, 44, 29-33. DOI 10.1007/s00127-008-0401-6.

Jiménez, A., Mondragón, L. y González-Forteza, C. (2007). Self-esteem, depressive symptomatology, and suicidal ideation in adolescents: results of three studies. Salud Mental, 30(5), 20-26.

Konick y Gutierrez (2005). Testing a model of suicide ideation in college students. Suicide and LifeThreatening Behavior, 35, 181 -192.

Lai, S. y Shek, D. (2009). Social Problem Solving, Family Functioning, and Suicidal Ideation Among Chinese Adolescents. In Hong Kong. Adolescence. 44. 391-406.

Mariño, M.C., Medina, M.E., Chaparro, J.J. y González, C. (1993). Confiabilidad y estructura factorial del CES-D en adolescentes mexicanos. 
Revista Mexicana de Psicología, 10, 141-145.

McLaren, S. y Challis, Ch. (2009). Resilience among men farmers: the protective roles of social support and sense of belonging in the depression-suicidal ideation relation. Death Studies, 33, 262-276. DOI: 10.1080/07481180802671985.

Miranda, I., Cubillas, M., Román, R. y Valdez, E. (2009). Ideación suicida en población escolarizada infantil: factores psicológicos asociados. Revista Salud Mental, 32, 495-502.

Moral, J., Sánchez, J.C. y Villarreal, M. (2010). Desarrollo de una escala multidimensional breve de ajuste escolar. REMA, 15(1), 1-11.

Musitu, G., Jiménez, T. y Estévez, E. (2009). Relaciones familiares y ajuste en el adolescente. Madrid: Instituto Ación Familiar.

Organización Mundial de la Salud (2000). Prevención del Suicidio. Un Instrumento para Médicos Generalistas, Ginebra, Suiza. Recuperado de: http://www.who.int/mental_health/media/general_p hysicians_spanish.pdf

Paneth, N. y Susser, M. (2002). The early history and development of the case-control study. Social \& Preventive Medicine, 47 (6), 359-365.

Pérez, B., Rivera, L., Atienzo, E., de Castro, F., Leyva, A. y Chávez, R. (2010). Prevalencia y factores asociados a la ideación e intento suicida en adolescentes de educación media superior de la República Mexicana. Salud Pública de México, 52(4), 324-333.

Perez, S. (1999). El suicidio, comportamiento y prevención. Revista cubana de medicina general, 15(2), 196-217.

Puentes, E., López, L. y Martínez, T. (2004). La mortalidad por suicidios: México 1990-200. Revista Panamericana de Salud Publica, 16(2), 102-109.

Radloff, L. S. (1977). The CES-D Scale: A self-report depression scale for research in the general population. Applied Psychological Measurement, 1, 385401.

Roberts, E. (1980). Reliability of the CES-D: Scale in different ethnic contexts. Psychiatry Research, 2, 125-134.

Rodrigo, M.J. (2009). Las competencias parentales en contextos de riesgo psicosocial. Intervención Psicosocial, 18, 113-120.

Rubini, M. y Pombeni, M.L. (1992). Cuestionario de conductas delictivas. Mimeo. Universidad de Bolonia. Facultad de Ciencias de la Educación.

Rudd, D. (1990). An integrative model of suicidal ideation. Suicide an Life-Threatening Behavior, 20, 16-30.
Sánchez-Sosa, J.C. (2009). Un Modelo explicativo de conducta alimentaria de riesgo en adolescentes escolarizados. (Tesis Doctoral. Universidad Autónoma de Nuevo León). Disponible en: http:// www.uv.es/lisis/gonzalo/tesis-rey.pdf

Sánchez-Sosa, J.C., Villarreal-González, M. y Musitu, G. (2010). Psicología y Desordenes Alimenticios. Un Modelo de Campo Psicosocial. Monterrey: UANL-UPO.

Smilkstein, G., Ashworth, C. y Montano, D. (1982). Validity and reliability of the Family APGAR as a test of family function. Journal Familiar Practice, 15, 303-311.

Suk, E., Van Mill, J., Vermeiren, R., Ruchkin, V., Schwab-Stone, M., Doreleijers, T. y Deboutte, D. (2009). Adolescent suicidal ideation: a comparison of incarcerated and school-based samples. European Child \& Adolescent Psychiatry, 18, 377383. DOI 10.1007/s00787-009-0740-1.

Sun, R., Hui, E. y Watkins, D. (2006). Towards a model of suicidal ideation for Hong Kong Chinese adolescents. Journal of adolescence, 29, 209-224.

Unikel, C., Bojórquez, I. y Carreño, S. (2004). Validación de un cuestionario breve para medir conductas alimentarias de riesgo. Revista Salud Pública, 46, 509-515.

Van Renen, L. y Wild, L. (2008) Family functioning and suicidal ideation/behaviour in adolescents: a pilot study. Journal of Child and Adolescent Mental Health, 20, 111-121.

Villalobos, F. (2009). Ideación suicida en jóvenes: formulación y validación del modelo integrador explicativo en estudiantes de educación secundaria y superior. (Tesis Doctoral. Universidad de Granada) Disponible en: http://hera.ugr.es/tesisugr/1805982x. pdf

Wilburn, V. y Smith, D. (2005). Stress, self-esteem and suicidal ideation in late adolescents. Adolescence, 40, 33-45.

World Health Organization (2001). The World Health Report 2001. Mental Health: New Understanding, New Hope. World Health Organization, Geneva, Suiza.

World Health Organization (2006). Suicide Prevention (SUPRE). New York, USA Recuperado de: http://www.who.int/mental_health/prevention/suicide/suicideprevent/en/.

Yoder, K. y Hoyt, D. (2005). Family economic pressure and adolescent suicidal ideation. Application of the family stress model. Suicide and Life-Threatening Behavior, 35, 251-264.

Manuscrito Recibido: 02/06/2010

Revisión Recibida: 08/09/2010

Manuscrito Aceptado: 18/10/2010 